\title{
Link Error impact on MPEG video over Wireless Broadband Networks
}

\author{
Javier Gomez-Castellanos \\ Columbia University, CTR \\ New York, NY 10027 \\ javierg@ctr.columbia.edu
}

\author{
Mahmoud Naghshineh \\ IBM Thomas J. Watson Research Center \\ Yorktown Heights, NY 10598 \\ mahmoud@watson.ibm.com
}

\begin{abstract}
This paper examines the transmission of MPEG video over high error rate links such wireless links. We present results showing the effect of transmission errors on MPEG streams and suggest an algorithm based on a combination of Forward Error Correction (FEC) and Automatic Repeat Request (ARQ) as a way to minimize its effects. We introduce a packet tagging technique that takes into account the particular semantic of MPEG flows to explicitly tell the network, both wired and wireless, which packets should necessarily be delivered correctly.
\end{abstract}

\section{Keywords}

MPEG, transmission errors, wireless links, FEC, ARQ

\section{INTRODUCTION}

The proliferation of portable computers with wireless capabilities together with the deployment of higher bandwidth wireless links is making it possible to extend video applications to wireless users. The Moving Picture Expert Group (MPEG) is becoming the standard video coding technique for most applications in the multimedia world. However, MPEG presents two big disadvantages for transmissions purposes over telecommunications networks. These problems are related with the periodic peaks in its bit rate due to the use of different compression techniques on consecutive pictures (Intraframe I pictures and Interframe $\mathrm{P}$ and $\mathrm{B}$ pictures) and also because of the difference in image quality perception of different types of pictures. In other words, the MPEG video stream is not constant in a quantitative way (bit rate), and neither in qualitative way (picture type). The inherent characteristics of wireless links, specially the higher error rates, will introduce a complete new set of issues that need to be taken into account in order to provide a reasonable QoS.

\section{ERROR RATE ANALYSIS}

So far, transmission of MPEG video streams has been studied over protocols that are designed with links of high reliability and where packet losses are mostly due to congestion. However, in wireless links packet loss is mainly a result of high bit error rate of the medium and this will drastically deteriorate received picture quality. In order to test the effect of wireless link errors on MPEG streams we used the following model:

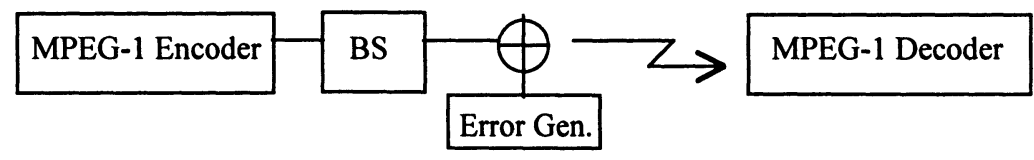

Figure 1. Wireless link error simulation testbed. 
In our test-bed, the original video frames are encoded using MPEG-1 and packetized into ATM cells (48 bytes). In order to isolate the error behavior of the system we assume that no contention for access on the wired link occurs and the necessary bandwidth in the network is always available to the video stream. The error generation module at the base-station uses an exponential error distribution to simulate errors over the wireless path. The video source we use is a MPEG-1 sequence of the film Ben-Hur with a Group Of Pictures (GOP) sequence IBBPBBPBBI. The bit rate of the video is $500 \mathrm{Kbps}$. In our experiments we test the two most common type of errors that occurs in wireless links. These types of errors are:

Fast fading: Fast fading causes the strength of the signal to vary rapidly with time. This may result in single bit random errors. For this source of errors and a bit error rates of $10^{-5}$, there is not a noticeable effect on the quality of the video at the receiver. Since most part of the encoded video stream is Discrete Cosine Transform coefficients (DCT), a DCT coefficient in error does not have a big impact on the overall quality of that particular group of pictures. However, for an error rate of $10^{-4}$ the situation changes drastically. For this error rate many control fields were corrupted by errors. These errors are enough to completely distort about 10 to 20 percent of the macroblocks per picture (refer to figures 2 and 3). When errors are introduced but just in the first bytes of the video stream, the whole session crashes and nothing appears on screen. The errors detected in the decoder happen to be related with information in the header of the video stream, which we refer to as control packets or control fields. The header contains the most important parameters for the MPEG decoder to work properly. As it is easy to understand control fields of the MPEG header should be strongly protected against link errors, and the strongest error correction care should be used here. In case that still some of the control fields were corrupted the decoder should ask for a retransmission.

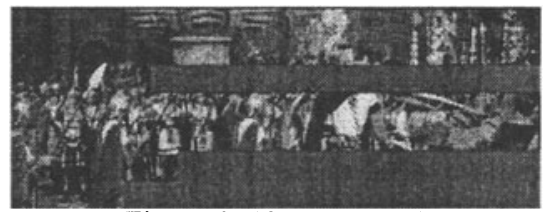

Figure 2. Picture 1 (I picture)

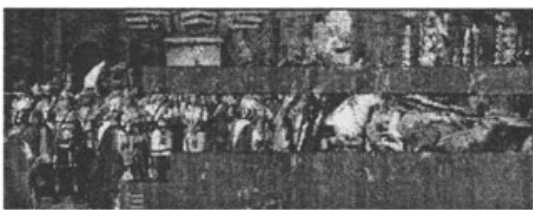

Figure 3. Picture 4 (P picture)

Results of these tests clearly suggest that it is the type of field in error, which for the most part decides the level of damage in the quality of video rather than simply the raw bit error rate. The problem now is that we need to protect control packets against transmission errors much more than others in order to achieve a reasonable quality of video at the mobile terminal.

Slow fading (shadowing). In the presence of slow fading the strength of the signal changes slowly with respect to time and this will cause bursts of errors. For this source of errors we assume that a entire cell can be corrupted even if Forward Error Correction data is attached to the packet. The main effects appearing in this case are mostly the same as in single bit random errors. The loss of a packet containing DCT coefficients has almost no effect on the overall quality of the video. However, the loss of a packet containing control fields will drastically deteriorate the quality.

Since different type of pictures will be delivered to the mobile terminal, it is important to understand how the video degrades when errors corrupt different type of pictures. For picture level tests we only introduce errors in a single picture to see the propagation effect of these errors in following pictures. In the first test we introduce errors but just in the first 
picture of the group of pictures (I picture). These errors will corrupt some macroblocks in that picture that remains during the following $\mathrm{P}$ and $\mathrm{B}$ pictures (refer to figure 3). The reason is that $\mathrm{P}$ and $\mathrm{B}$ pictures are decoded with respect to the previous $\mathrm{I}$ or $\mathrm{P}$ pictures. This issue makes it imperative to protect I pictures with a stronger FEC algorithm or by using retransmissions. If the I picture is completely corrupted it is no sense to transmit the rest of the group of pictures, since all the information in the remaining pictures will be useless at the mobile terminal.

In the second test, errors do not corrupt packets in I pictures, but errors will corrupt $\mathrm{P}$ pictures. Common errors we got are mostly DCT coefficients out of range that introduce dark spots on the screen that in some sense may be tolerable. However, protecting I pictures against link errors improve the quality of the video noticeably. Some times dark macroblocks appear and remain on the following pictures if errors hit $\mathrm{P}$ pictures macroblocks that are used as reference by the followings $P$ and $B$ pictures (figure 4). This suggests that important control fields of $P$ pictures should be also guaranteed an error-free delivery.

Since B pictures need one of the previous pictures and one of the following pictures to be decoded they require out of order transmission. For these reasons B pictures are not suitable for real time applications. Therefore a sequence of MPEG may have only intraframes pictures (I I I I I I I I) or may contain intraframe/interframe pictures (I P P P P P P P I). In a mixed Intraframe/interframe scenario except for the first picture, the remaining pictures ( $P$ pictures) need the previous picture to be decoded. This means that if errors hit one picture macroblock in the $i$ th. picture, that error will also appear in picture $i+1, i+2, i+3 \ldots N-i$, where $\mathrm{N}$ is the number of pictures in the group of pictures. As a result, one macroblock in error is going to remain on the screen until a new I picture arrives to the decoder. Knowing this, the mobile terminal can, if necessary, request the encoder to increase the ratio of intraframe to interframe pictures so corrupted blocks will just propagate a maximum of $\mathrm{N}-1$ pictures.

Reducing the number of $\mathrm{P}$ pictures among I pictures will noticeably increase the bit rate but this can be compensated with a bigger step size when quantization is taken place at the encoder. In the case that bandwidth is still a limitation to support more I pictures, the encoder might even reduce the picture rate to compensate for the additional I pictures bit rate. The basic design criteria will be that it is better to show few good pictures per second in the mobile terminal rather than show many bad pictures.

Picture.Type:

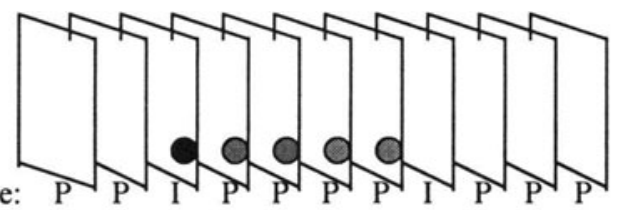

Due to link errors

Decoded erroneously

Figure 4. Propagation of errors in a GOP

Another way to overcome slow fading situations will require the source to slow down its transmission rate (say reduce the picture rate) so the Bit Error Rate (BER) decreases to a point where FEC alone is again enough to kept and acceptable video quality. However, the time involved in telling the source to decrease the bit rate and the time the mobile terminal can start seeing the effects will take to long. For these reasons we consider that an ARQ capability should still remain to allow retransmission of important packets that might 
become lost in the air. This leads to a hybrid FEC/ARQ error correction protocol to overcome single and packet level errors

Because of the potential long time propagation delay involved, we propose to snoop the connection at the base station so the retransmission times are much shorter allowing ARQ protocols to be more effective. This implementation makes it necessary to buffer the MPEG packets at the base station until acknowledgments are received from the mobile terminal. However, as we mentioned in the previous discussion, not all the packets need to be forcefully received correctly. In this case we propose to mark important control packets with a flag that guarantees that those packets will be delivered correctly (packets that may be buffered and re-transmitted if necessary). This can be done by increasing even more the FEC error capacity or/and by retransmissions [4]. Other packets (mainly packets with DCT coefficients) will not be tagged with the flag.

Marking each packet with a flag (potentially more than one bit) makes the network understand the semantic of MPEG in order to maximize the quality of the video at the mobile terminal even if congestion and transmission errors occurs. The penalty is that now the application layer at the encoder should be modified to send priority information to the transport layer in order to set the flag in each packet. At the same time routers along the path (at least the base-station) should look inside the packet to read and process the flag.

For picture level errors we propose to mark all the packets belonging to I pictures so that these packets will reach the mobile correctly with an acceptable high probability. It also has to be guaranteed that packets containing important fields of $P$ pictures reach the mobile correctly. Finally, depending of the error rate behavior at any particular time, we may dynamically increase or reduce the number of $P$ pictures between I pictures.

\section{CONCLUSIONS}

We showed the effects of high error rates on MPEG streams pointing out some ways to compensate for these errors. Also, we show that for the most part, it is the type of field in error which determines the level of damage to the quality of the video rather than the raw bit error rate only. It is important to preserve an ARQ capability at the base-station in combination with common FEC techniques as a way to compensate for both, the high error rate characteristics of the links and the impact in image quality of different type of pictures. We also discussed the advantages of periodically sending feedback information to the source to readapt to changes in the network.

\section{REFERENCES}

[1] Netravaly A. and Hanskell H. (1994) Digital Pictures, Plenum, second edition.

[2] Pancha P and El Zarki M. (1995) A look at the MPEG video coding standard for variable bit rate video transmission, INFOCOM, pp. 85-92.

[3] Moura J., Jaaiinschi R. S., Shiojiri H. and Jyh-Chering Lin (1996) Video over Wireless, IEEE personal Communications, february, pp. 44-54.

[4] Naghshineh M., Schwartz M. and Acampora A. (1996) Issues in wireless access broadband networks. Wireless Information Networks Academic Publishers.

[5] Karlson G. (1996) Asynchronous Transfer of Video, IEEE comm. magazine, august. 